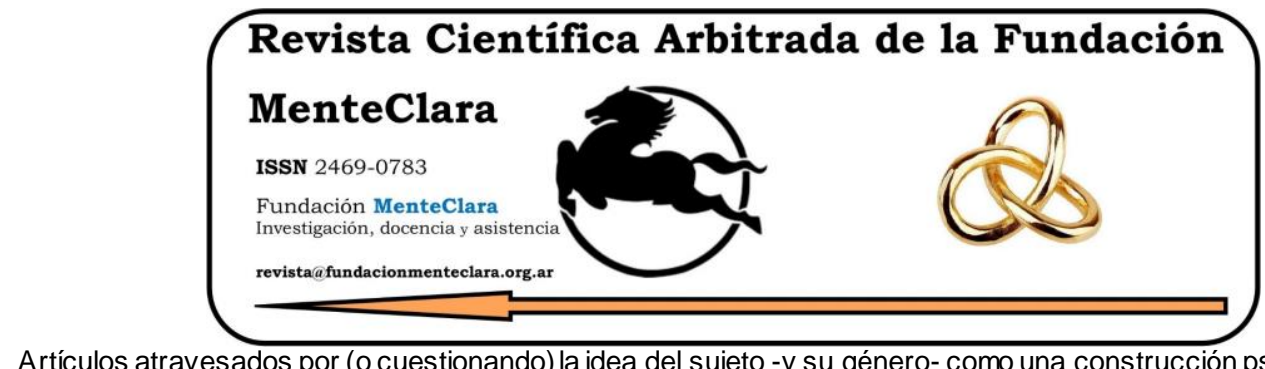

Artículos atravesados por (o cuestionando) la idea del sujeto -y su género- como una construcción psicobiológica de la cultura. Articles driven by (or questioning) the idea of the subject -and their gender- as a cultural psychobiological construction.

Vol. 7 (2022), enero-diciembre ISSN 2469-0783

https: / / datahub.io/dataset/2022-7-e270

\title{
ESTRATEGIAS DIDÁCTICAS MEDIADAS POR LAS TECNOLOGÍAS DE LA INFORMACIÓN Y LA COMUNICACIÓN (TIC) EN ESTUDIANTES DE NIVEL PRIMARIO CON NECESIDADES EDUCATIVAS ESPECIALES. EXPERIENCIAS EN EDUCACIÓN ESPECIAL
}

\author{
DIDACTIC STRATEGIES MEDIATED BY INFORMATION AND COMMUNICATION \\ TECHNOLOGIES (ICT) IN PRIMARY SCHOOL STUDENTS WITH SPECIAL \\ EDUCATIONAL NEEDS. SPECIAL EDUCATION EXPERIENCES
}

Maria Cecilia Roma MariaCecilia.Roma@uai.edu.ar
Universidad Abierta Interamericana.

Cómo citar este artículo / Citation: Roma, M. C. (2022). Estrategias didácticas mediadas por las tecnologias de la información y la comunicación (TIC) en estudiantes de nivel primario con necesidades educativas e speciales. Experiencias en educación especial . Revista Científica Arbitrada de la Fundación MenteClara, Vol. 7 (270). DOI:

https://doi.org/ 10.32351/rca.v7.270

Copyright: (C) 2022 RCAFMC. Este artículo de acceso abierto es distribuido bajo los términos de la licencia Creative Commons Attribution 4.0 International License (CC BY 4.0). Recibido: 08/02/2022. Aceptado: 12/02/2022 Publicación online: 14/02/2022

Conflicto de intereses: Ninguno que declarar.

\section{Resumen}

El artículo describe la investigación realizada por esta autora con el fin de analizar estrategias didácticas mediadas por las tecnologías de la información y la comunicación (TIC) en el ámbito de las escuelas de educación especial, de nivel primario, de gestión pública, dependientes del Ministerio de Educación de la Ciudad de Buenos Aires. Los hallazgos de la investigación dan cuenta principalmente del potencial de las estrategias didácticas mediadas por TIC que los y las docentes despliegan, dentro y fuera del aula, para estimular y favorecer el aprendizaje de estudiantes con diversas problemáticas en educación especial. 


\begin{abstract}
This article describes the research realized by this author for analyze. didactic strategies mediated by information and communication technologies (ICT) in the context of special education schools, primary level, public management, dependent on the Ministry of Education of the City of Buenos Aires, are analyzed. The research findings mainly for the potential of ICT-mediated teaching strategies that teachers deploy to stimulate and promote the learning of students with various problems in special education.
\end{abstract}

Palabras Claves: TIC; Educación Especial; Recursos educativos digitales; Educación primaria

Keyw ords: ICT; Special Education; Digital Educational Re sources; Primary Education 


\section{Introducción}

La posibilidad de abordar actividades o propuestas promovidas por las tecnologías en alumnos con necesidades especiales o con problemas de aprendizaje estimula a que el mismo niño pueda encontrar su propio ritmo de trabajo y de producción. En caso que el docente esté en condiciones de asistir a este alumno, podría ofrecerle propuestas pedagógicas más o menos complejas que potencialicen el aprendizaje según las capacidades del niño. Para esto es imprescindible que el docente pueda pensar construcciones didácticas pertinentes. Es decir, depende del docente, de su preparación y de la calidad de su intervención al momento de repensar una enseñanza diferenciada y poderosa, esto le brinda al niño propuestas más creativas y lúdicas favoreciendo así su predisposición a la actividad. La opción de abordar apropiadamente este proceso abre la puerta a cambios profundos en la calidad de las propuestas pedagógicas ofrecidas por el equipo docente (Maggio, 2012). También es fundamental pensar que existe un antes, durante y un después en la implementación de estas propuestas. El antes implica que el docente piense y construya un modelo de práctica según los intereses, estímulos y posibilidades del grupo, de este modo, delimita el tipo de secuencia didáctica posible. En la instancia de implementación debería haber un análisis del proceso en función de los resultados que se vayan evidenciando con la práctica. En la instancia posterior se debiera pensar en una evaluación o reflexión profunda sobre los resultados finales, considerando el andamiaje ofrecido en el acompañamiento al alumno relacionado con el marco conceptual que el docente establece en una primera instancia y pensando en futuras prácticas (Maggio, op.cit).

Teniendo en cuenta que actualmente las prácticas educativas pueden considerarse un desafio, entonces es posible ser partícipes de la construcción de criterios disciplinares y todavía fuera de lo disciplinar, 
es decir, extensivo al hogary la vida diaria. Al ser implementadas las TIC como sostenedoras de prácticas colaborativas a través de la red, se posibilitan amplias opciones de atender a la diversidad cultural y a la diversidad de identidades locales desde las propuestas educativas (Soletic en Litwin, 2009). En este contexto es fundamental explorar las capacidades, habilidades y competencias de los/as estudiantes para llevar al máximo su potencial de desarrollo de conocimiento. Enseñar a construir razonamiento crítico, analizar y resolver problemas, establecer relaciones y generar producciones significativas para el niño es el eje central de la implementación de las TIC en el proceso de enseñanza aprendizaje (Maggio, 2012).

\section{Cuestionamientos iniciales}

Luego de haber realizado una introducción al tema que nos ocupa, surgen algunas preguntas que se intentarán responder a lo largo del artículo: ¿El uso de las TIC en los procesos de enseñanza para alumnos y alumnas con discapacidad intelectual o problemáticas similares en las escuelas primarias puede favorecer su aprendizaje? Dentro de este marco se generan otros diversos cuestionamientos: ¿el uso de estrategias didácticas mediadas por TIC realmente facilitan el aprendizaje de los niños y de las niñas con discapacidad intelectual o problemáticas similares? ¿Puede el uso de las TIC optimizar el aprendizaje de estos niños específicamente en la enseñanza de los contenidos donde la experimentación y la abstracción son procesos elementales para la integración del nuevo conocimiento? ¿El uso de las TIC en alumnos con necesidades especiales puede colaborar en la comprensión de conceptos más abstractos? ¿Posibilitan una construcción dual de conocimiento (docente-alumno) a partir de los intereses de los niños? ¿Los alumnos 
manifiestan menos resistencia al aprendizaje cuando las estrategias están mediadas por TIC?

Durante el artículo se espera responder algunos de estos interrogantes y además abrir caminos para que otros investigadores con interés en la temática puedan indagar estos cuestionamientos.

\section{Las TIC en la educación actual}

Las Tecnologias de la Información (TI) hacen referencia a la implementación de los recursos necesarios como la microelectrónica, las telecomunicaciones, los programas informáticos y las redes para manipular la información, es decir, para convertirla, almacenarla, administrarla, transmitirla y recuperarla (Liguori, 2000). Otro punto de vista, no muy diferente es el correspondiente a Buckingham (2012) quien piensa las tecnologías digitales a modo de medios de comunicación, es decir, permiten la comunicación entre personas y además son representativas del mundo. Al representar el mundo, Internet, las redes sociales, los juegos virtuales, se transforman en elementos sociales y por lo tanto también son culturales. El mismo autor hace referencia a la alfabetización digital en el sentido de considerarvarios aspectos, además de la lectura crítica, el niño, el joven y todo alumno en general debería poder producir y escribir en los medios su propio material (Buckingham, 2006). Así mismo sostiene que el uso de Internet en los hogares colabora con el desarrollo de la propia autonomía y autoridad como usuarios de la red. Estas capacidades son justamente las cualidades que se deberian ayudar a desenvolver en las escuelas desafiando el aprendizaje pasivo y receptivo.

Todos estos elementos constituyen un nuevo escenario que ha transformado tanto el entorno educativo como el social. Este contexto 
obliga a resignificar la práctica educativa considerando nuevas formas de aprender y de enseñar. Como menciona César Coll (2008) la educación se define como "tecnología de la información y de la comunicación, la primera para transformarla en conocimiento y la segunda para ayudar a los que aprenden a transformar esa información en conocimiento". Por tanto, el proceso educativo se encuentra en una transformación innovadora con nuevos recursos y herramientas. Este espacio permite un nuevo escenario de comunicación e información así como también permite y desarrolla un nuevo modo de interacción, entretenimiento, expresión de emociones y sentimientos, es decir, de desarrollo social (Echeverría, 2000). También Sánchez Montoya (2007) sostiene que los paradigmas emergentes tecnológicos potencian la calidad de los aprendizajes del alumno con discapacidad desde el momento que los nuevos modelos pedagógicos son más interaccionistas, no solamente desde las tecnologías de apoyo sino desde los programas que los alumnos pueden implementar como recursos optimizadores de sus aprendizajes.

Las TIC también proveyeron un modelo colaborativo que implica un cambio de conceptos entre el alumno y la tecnología modificando el sentido del uso de la web en la escuela. Se posibilita de este modo, la idea de que el uso de las TIC en el trabajo del aula podría favorecer un residuo cognitivo como sobra residual en forma de capacidades y habilidades que serian transferibles a otras situaciones problemas abordadas en la currícula escolar (Lion, 2006).

Estas tecnologias además han permitido favorecer el desarrollo cognitivo en niños con discapacidad intelectual y/o necesidades educativas especiales desde el momento en que han permitido el desarrollo de los aspectos comunicacionales, expresivos, de habilidades y perceptivos. Es decir, son metodologias que les favorece a los alumnos 
la posibilidad de desvelar y apropiarse de nuevos conceptos propios de las diferentes áreas de conocimientos (Castellano, 2011).

\section{Materiales y métodos}

Para esta investigación se estudiaron las estrategias didácticas que implementaron los y las docentes de 3 escuelas de la Ciudad de Buenos Aires, 1 escuela para niños con trastornos emocionales severos, 1 escuela integral interdisciplinaria y 1 escuela para alumnos con discapacidad intelectual, quienes aportaron sus experiencias de aula. También se consideró que los alumnos sean de alrededor de quinto grado de escuela común o grupo en edad similar. El criterio para la selección de estos casos respondió a que en la edad de entre 11 y 12 años aproximadamente comienza el desarrollo de las operaciones formales, del pensamiento abstracto (Piaget, 2005). Dado que en estos niños el desarrollo de este tipo de razonamiento es más dificultoso, es que se propuso la estrategia mediada por TIC para favorecer el aprendizaje.

Se eligió para el análisis, el método de la comparación constante (Glaser y Strauss, 1967) porque al tratarse de una investigación con preponderancia de abordaje cualitativo, nos permitió abordar el im pacto de las prácticas docentes en el contexto escolar durante la implementación de estrategias con TIC, durante el mismo momento en que el docente fue experimentando su aplicación para mejorar el proceso de aprendizaje de alumnos con dificultades o discapacidad intelectual. Este método integra al investigador en interacción con la dinámica del aula. Permite realizar un registro observacional y analítico en relación al contexto. Sin embargo se percibió la necesidad de enriquecer la investigación con encuestas a modo de sistematizar datos cuantitativos tales como qué cantidad de docentes incluyen las tic en sus propuestas 
didácticas, cuáles son los recursos digitales que el docente utiliza habitualmente, en qué momento de la jornada escolar los alumnos utilizan las computadoras y otras indagaciones relevantes para este proyecto

A continuación se detallarán algunos de los proyectos elaborados en las escuelas según las posibilidades de cada población y su análisis considerando las posibilidades de producción de alumnos.

\section{Vincul-Arte}

En el 2015, se ha llevado a cabo el proyecto "Vincul-Arte a un clic" elaborado por la una escuela de educación especial de niños con discapacidad intelectual. El proyecto presentó la vinculación entre el arte y las TIC. El objetivo fue abordar las lecturas. Se fundamentó en la defensa de una escuela inclusiva en la cual todos los alumnos pudieran ser beneficiarios de una enseñanza repensada según sus necesidades. Siendo así, se planteó la necesidad de reflexionar sobre la práctica docente, ofreciéndole a los mismos desafios que le permitan elaborar estrategias pedagógicas alternativas. En el marco de las múltiples alfabetizaciones se hace centro el acto de leer y escribir para comunicarse y las tecnologías son el recurso que les ofrece, además, la interactividad, lo que resulta atractivo para esta población (Angeloff, Muratore, Campo y Savo, 2015). Este proyecto permitió abrir el próximo camino que es la utilización de las redes sociales educativas. El objetivo fue promover el intercambio entre las dos escuelas, la escuela de educación especial y una escuela primaria común, ambas pertenecientes al mismo distrito escolar. El proyecto propuso Edmodo como red social para promover la lectura y escritura entre los alumnos además del intercambio social entre 
ellos. Las actividades realizadas en la propuesta de Vincul-arte se registraron y se compartieron por Edmodo (Savo, Muratore, 2015).

\section{El blog escolar}

El blog es otro recurso rico en propuestas para la lectura y escritura. En 2014 se inició el proyecto "Blog escolar" en otra de las escuelas en la cual además de alguna problemática cognitiva se presentaban problemáticas de tipo emocionales. El blog se elaboró como una propuesta propulsora de las competencias comunicativas ofreciendo los diferentes formatos, imagen, audio y narración. De este modo se estimulaba al alumnado a manifestar intereses, experiencias, situaciones emocionales y demás. Se creó una cuenta de correo y a partir de esta cuenta cada grupo creó su blog con las características particulares del grupo (Roma, 2014).

De este modo se consiguió que los alumnos se animaran a escribir y a compartir sus producciones con sus compañeros. El poder narrar sus vivencias del fin de semana por ejemplo resultaba complejo pues los recursos expresivos conscientes eran escasos. A través del blog pudieron por lo menos compartir una foto representativa de su fin de semana, en otros casos a pesar que la coherencia en la secuencia de hechos también presenta dificultades, hubo narración escrita.

\section{La historieta}

Otro recurso ampliamente enriquecedor para promover el desarrollo de la lectura y escritura es la historieta en cuadritos o cartoons. Con la misma población se realizó en 2013 el proyecto "La historieta: una posibilidad de comunicación y representación” (Roma, 2013). Son variados los recursos para crear historietas, tanto en linea como sin 
conexión. El objetivo era promover la narración a partir de un personaje de interés para el alumno. Los estudiantes lograron representar a través de una secuencia narrativa una situación conflictiva significativa para cada uno de ellos, aún con las dificultades que esto les representaba.

\section{E1 texto biográfico}

En otro establecimiento educativo se abordó el texto biográfico. El objetivo continúa siendo la lectura y la escritura. Para estimular al alumno la propuesta se centró en una personalidad de interés para el niño. En este caso, la experiencia se realizó con un alumno en forma individual. Se trabajó con búsquedas en la red, seleccionando información relevante e imágenes acorde al texto. El recurso utilizado fue el editor de presentaciones que era un recurso que el niño ya había usado en otro momento. Como el objetivo se centraba en la investigación y escritura no se propuso otro recurso nuevo para el alumno para que no representara un motivo de frustración y desestímulo. El niño logró investigar la vida del deportista seleccionado y narrar sintéticamente su historia (Roma, 2015), indagando inclusive la localización geográfica del lugar de nacimiento y el club para el cual jugaba.

\section{Cuentos de terror}

Continuando con la producción literaria, se llevó a cabo un proyecto de escritura creativa con alumnos de 5 to y 6 to grado de escuela integral interdisciplinaria. Son alumnos que habiendo concluido 5to grado necesitaban un tiempo de preparación para ingresar a 6to grado (Baldissera, 2016) (Roma, 2014). La idea de realizar cuentos de terror surgió de los propios alumnos a partir de una actividad de radio que estaban trabajando. Era un grupo que presentaba dificultades para ser 
convocados para una temática además de labilidad atencional, dificultad en la lectura y expresión oral. A pesar de esto eran constantes en concluir con las propuestas que iniciaban. En este caso todo el grupo elaboró en conjunto una historia de terror implementando también el editor de presentaciones. Los alumnos no solamente narraron la historia sino que le incorporaron audios propios para cada situación. En este caso el contenido central fue recontar y contar cuentos (Diseño Curricular de 2 do ciclo, 2004).

\section{Federico se hizo pis}

La siguiente propuesta fue elaborada por niños que presentan retraso madurativo, Sindrome de Down y Trastorno generalizado del Desarrollo. El proyecto tuvo como propósito que los/as niños/as desarrollen la escucha atenta, lean, cuenten, disfruten y recreen diversas clases de historias y relatos, para que se vinculen con la literatura y al mismo tiempo desarrollen aspectos de las prácticas del lenguaje. Dentro de las características cognitivas del grupo se destacaban la copia de palabras y el reconocimiento de letras. En algunos casos se lograba la escritura espontánea, y la lectura de textos cortos. En esta ocasión se realizó la recreación del cuento "Federico se hizo pis" de Graciela Montes, con objetivo de que el grupo más avanzado se lo pueda narrar al grupo de educación inicial. Se tomaron como base los diseños realizados por el primer grupo mencionado y se trabajó en el relato oral de los mismos niños. Era un grupo con serias dificultades expresivas a nivel oral por tal motivo resultó un logro significativo que realicen una grabación narrada oralmente.

Es posible observar en estas propuestas el enfoque central sobre aquello en lo que el sujeto puede producir, es decir, el eje está puesto en 
la potencialidad del alumno (Fainblum, 2004). En estos ejemplos de prácticas pedagógicas se buscó articular aprendizajes relevantes para la comprensión y organización de la realidad, partiendo de la demanda del alumno, haciendo cosas con sentido. De este modo, también se desarrolla un lugar docente en donde su intervención pedagógica puede promover efectos terapéuticos. Un aprendizaje en estas condiciones produce repercusiones en la estructura psíquica del alumno haciendo que el proceso educativo se desempeñe como elemento estructurante del psiquismo (DGCyE, 2008). Así, como resultado de estas prácticas las consecuencias del proceso de aprendizaje escolar terminan extrapolando la mera construcción de conocimientos, implicando el desarrollo de otros conceptos como el de la transferencia o rasgos identificatorios en los cuales la escena didáctica se amplía más allá de cualquier planificación (DGCyE, 2008).

\section{Resultados}

Las encuestas aplicadas a los 6 docentes participantes de la experiencia, vinieron a registrar y ofrecer datos concretos sobre las prácticas de los maestros desde la perspectiva de los mismos, permitiendo de este modo verificar la coherencia entre los discursos y la acción del docente en el aula. Por ejemplo, las encuestas han evidenciado que solamente el $33,33 \%$ de los maestros utilizan las computadoras de forma destacada en sus propuestas didácticas anuales y el 50\% considera las computadoras de relevancia secundaria para sus planificaciones anuales. 


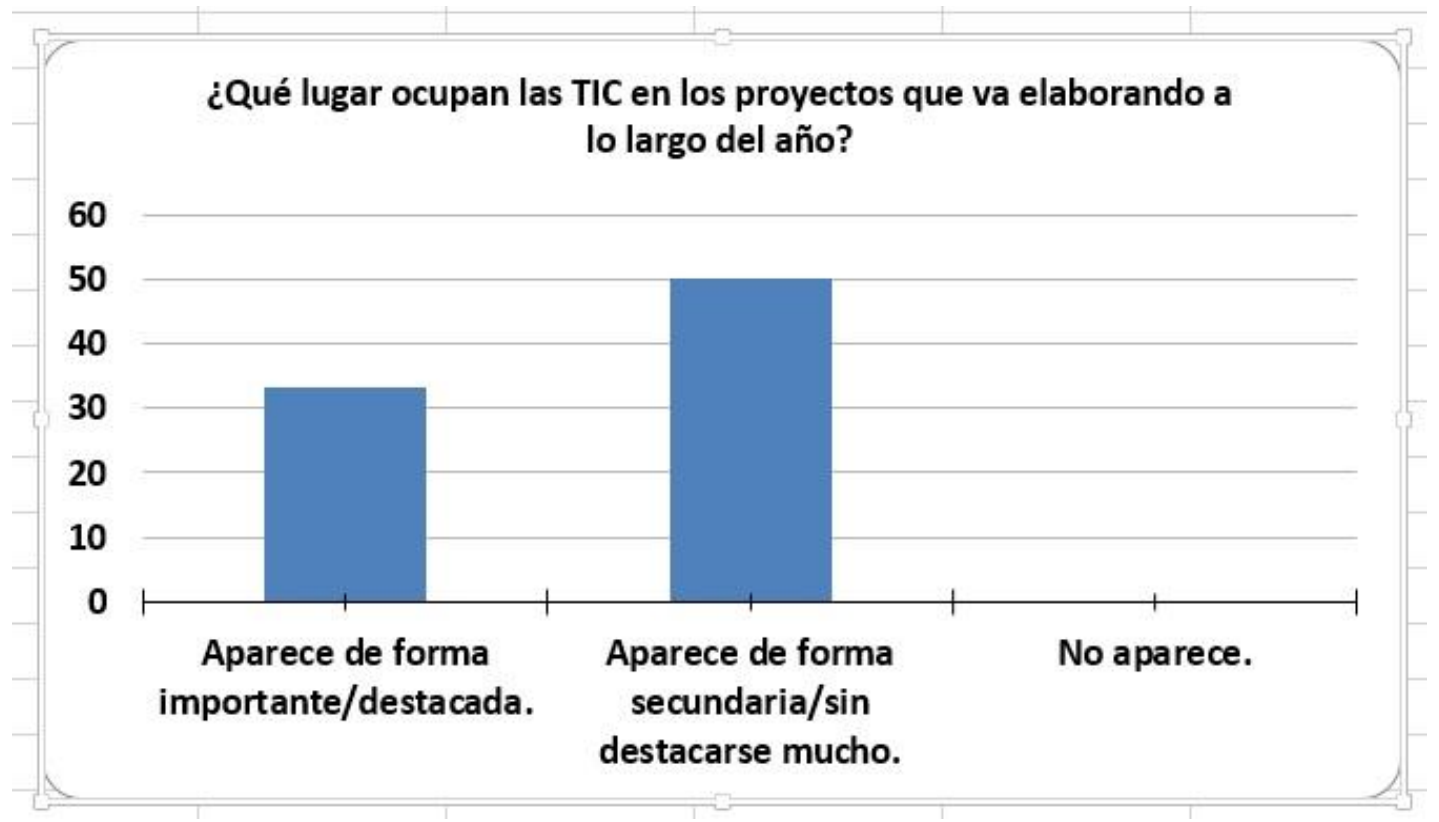

Fuente: (Roma, 2018)

Otro aspecto importante es que los recursos más usados son el editor de texto y el editor de audio, con un 83,33\% de impacto, pero otros recursos como la búsqueda con navegadores ocupan el 33,33\% de uso como recurso educativo, abriendo la posibilidad de indagatoria sobre la búsqueda de información o la posibilidad de investigación que se le ofrecen al alumno.

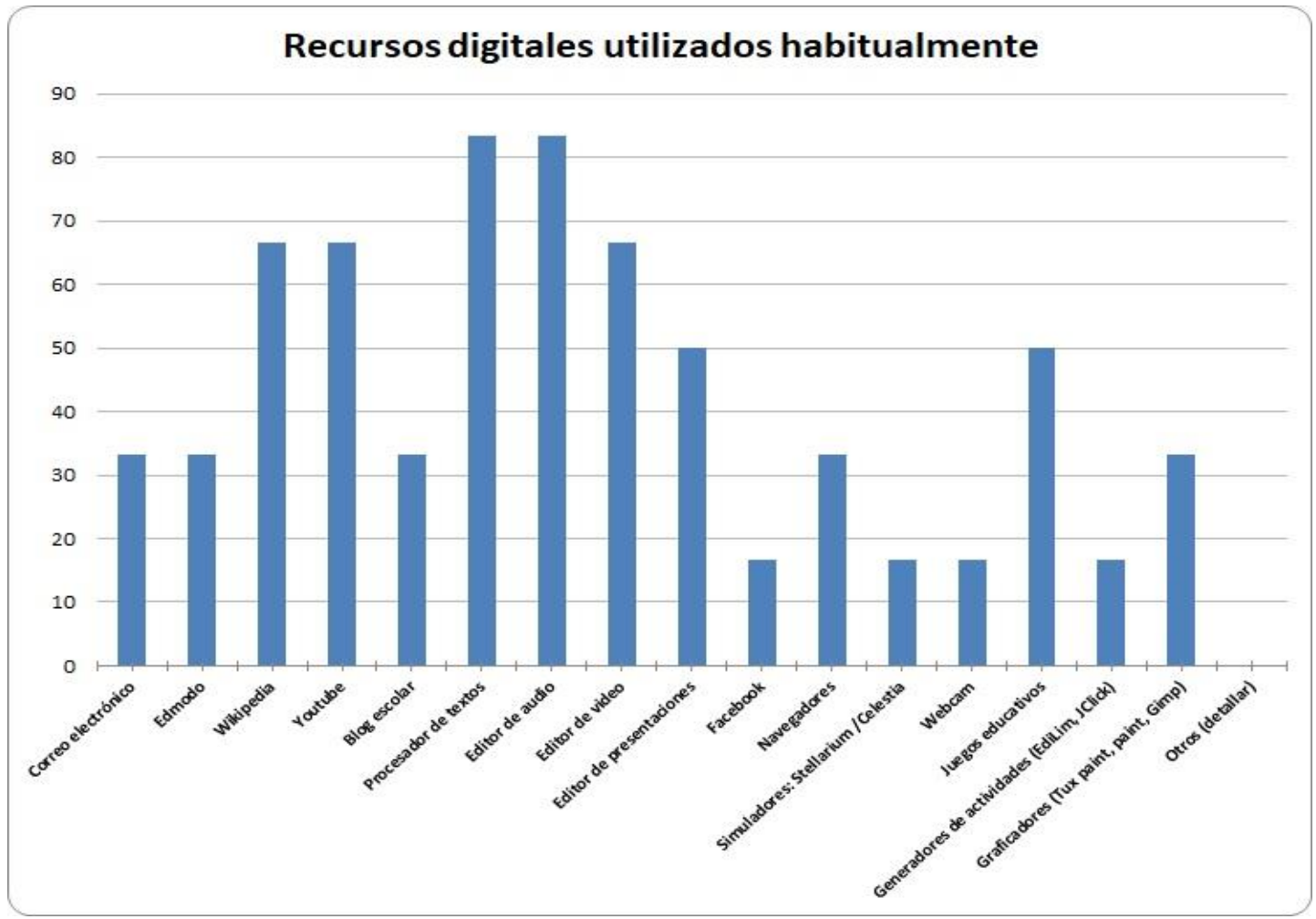


Sin embargo, los docentes también registraron que el mayor uso que se le hacen a los recursos digitales se realizan en el aula con un $83,33 \%$ de respuesta, en detrimento del uso en otros espacios lo que podría indicar un uso destacadamente pedagógico.

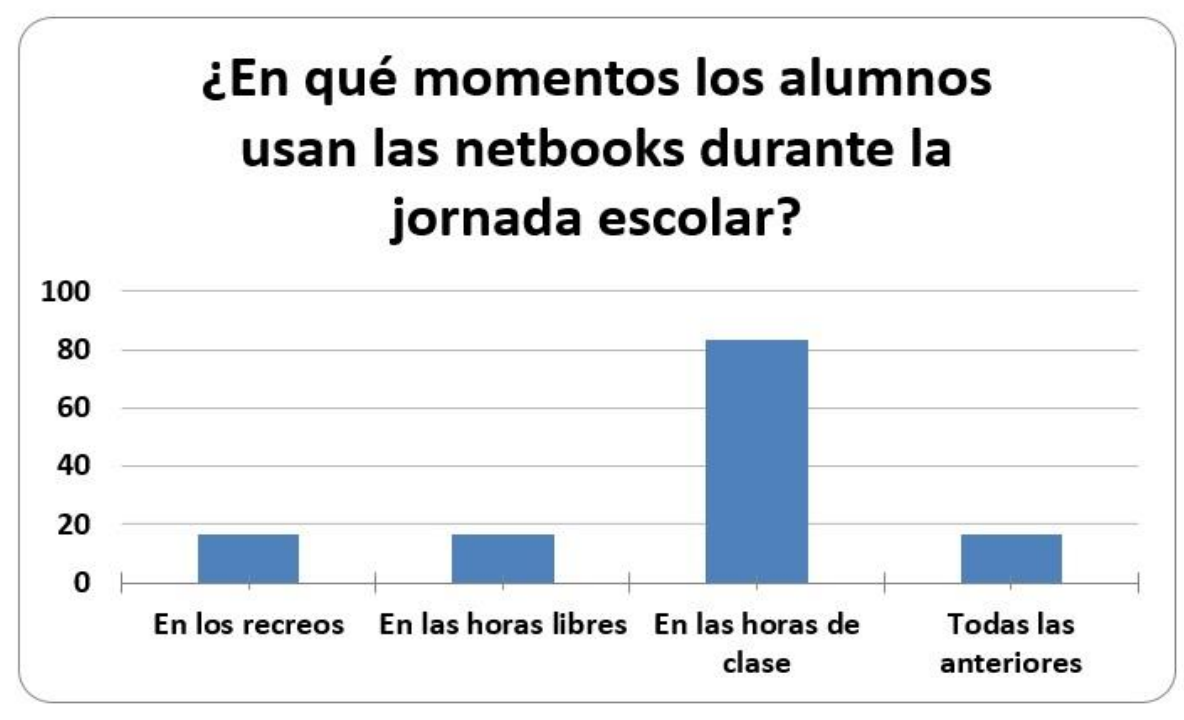

Fuente: (Roma, 2018)

La encuesta realizada ha pretendido, al mismo tiempo, corroborar la efectividad del trabajo mediado por las TIC desde la perspectiva de los propios alumnos observando el logro de un aprendizaje significativo.

De este modo una mediación triangulada buscó probar diferentes valores de un mismo fenómeno para alcanzar la mayor exactitud al ser un abordaje que permite variados puntos de vista. De este modo se define su validez (De Gialdino, 1992).

A partir de los datos obtenidos por medio de los diversos métodos implementados y de su triangulación fue posible realizar un análisis crítico sobre aquellas estrategias que favorecen la transferencia y la apropiación significativa de los contenidos curriculares atravesados tecnológicamente. 


\section{Discusión y conclusiones}

En la modalidad de Educación Especial el aprendizaje suele exigir una atención pedagógica individualizada. Los docentes plantean que se centran en los contenidos correspondientes al ciclo, los saberes previos de los/as estudiantes y además identifican aquellos recursos que permiten estimular la creatividad en los niños. Destacan el intercambio con los/as alumnos/as para seleccionar un recurso y actividad con ese recurso que sea de relevancia y significatividad para el grupo. Pues, es fundamental responder a las diversidades de todos los estudiantes y llevarlos hacia una mayor participación en sus respectivos aprendizajes. Es por esto que llegar a un consenso no es fácil. El docente conversa, identifica los intereses de los niños y cruza los contenidos que pueden abordarse a partir de ese interés.

El uso de las TIC en los procesos de enseñanza para alumnos/as con las dificultades detalladas en las escuelas primarias, favorece el aprendizaje desde el momento que la escuela promueve al desarrollo de capacidades y competencias que permitan la apropiación de contenidos generales y específicos de la cultura, necesarios para que los alumnos puedan ser ciudadanos activos en su contexto sociocultural de referencia. Para esto, la escuela buscaría lograr un determinado equilibrio al ofrecer una respuesta educativa que comprenda ese contexto y al mismo tiempo que sea diversificada para dar respuesta a ese entorno sociocultural en común a todos. Esta respuesta evita la discriminación y la desigualdad de oportunidades respetando las características y necesidades individuales. Las necesidades educativas son comunes a todos los alumnos, en relación con su desarrollo personal y socialización, expresados en el currículum escolar. Paralelamente se hace indispensable considerar que no todos los alumnos y alumnas presentan el mismo bagaje de experiencias previas y de aprendizajes establecidos 
en el currículum. Cada niño/a presenta competencias, habilidades, intereses, ritmos, capacidades, motivaciones y experiencias diferentes que intermedian su proceso de aprendizaje, siendo único en cada caso. Las experiencias pedagógicas destacadas demuestran que han favorecido la adquisición de saberes por parte de los alumnos. El enfoque en este caso ha sido el uso de los recursos digitales que hace el o la docente en sus propuestas didácticas. Por ejemplo, en el caso del planteo del lugar que ocupan las TIC en la planificación anual de los docentes las respuestas han manifestado que aparece de forma destacada en el 33, $33 \%$ de los casos y en un segundo plano en un 50\% de los casos dejando en evidencia el avance que ha representado el uso de las tecnologias en el proceso de enseñanza-aprendizaje de los alumnos en cuestión.

También se ha manifestado la posición favorable al reflexionar con relación al uso de estrategias didácticas mediadas por TIC considerando que efectivamente facilitan el aprendizaje de los niños con discapacidad intelectual u otras necesidades educativas especiales. Sobre este aspecto se ha expresado a lo largo del presente artículo la relevancia que presentan los intereses de los alumnos al momento de construir las propuestas pedagógicas junto a ellos. Los ejemplos de las actividades por medio de los diálogos y registros visuales dan cuento de esto.

Se ha manifestado durante el texto que el uso de las tecnologias optimizan el aprendizaje de estos niños al ofrecerles la posibilidad del juego a través de la experimentación, de este modo se fomenta la posibilidad de abstracción como proceso elemental para la inte gración de nuevos conocimientos.

Los contenidos se abordan posibilitando una construcción dual de conocimiento docente-estudiante a partir de sus intereses. De este modo el docente se asegura que las propuestas puedan ser continuadas en el 
tiempo favoreciendo la conclusión de los proyectos. La co-construcción de conocimiento favoreciendo un aprendizaje participativo y colaborativo se ha manifestado relevante para favorecer una postura receptiva en los alumnos y así promover el desarrollo de los contenidos académicos.

Algunas necesidades especiales pueden resolverse con acciones tales como flexibilizar los tiempos en el abordaje y comprensión de determinados contenidos, adecuar las estrategias buscando la óptima para ese alumno, usar materiales educativos más atractivos y sintéticos, diseñar actividades más ricas y variadas o fomentar el trabajo colaborativo. En otros casos se hace necesario repensar ayudas, recursos y secuencias didácticas especiales con un trabajo más individualizado (Guijarro, 1990).

Después del análisis efectuado a través de las respuestas de los docentes y de los intercambios con los mismos también es posible decir que el lugar de las TIC todavía necesita abrirse paso. Es un proceso que está instalándose en el ámbito educativo, pues en el ámbito social y cultural ya está instalado.

César Coll menciona que todavía el proceso de transformación y posibilidades de mejora que proveen las TIC se deben entender como un potencial plausible de hacerse realidad o no. Cuando se habla de hacerse realidad se hace referencia al contexto en el que las tecnologías son efectivamente aplicadas (Coll, 2008).

El aprendizaje en este sentido se abre al entorno, comprendiendo que no siempre es un entorno formal. Puede ser informal, darse en cualquier momento, planificado, no planificado, se comparte entre pares, entre familias, entre colegas. Es importante que el docente considere este aspecto al momento de planificar con TIC (Grané, 2011). En una escuela inclusiva estas consideraciones adquieren relevancia redoblada pues es 
necesario repensar el contexto en torno a la ubicuidad, universalidad e invisibilidad de las tecnologias. En este ámbito los/as alumnos/as se benefician de una enseñanza adaptada a sus necesidades. Replantear la práctica docente proponiendo estrategias pedagógicas diversas para la construcción de:

* aprendizajes desde las multialfabetizaciones,

* aprendizajes constructivos,

* la valoración de las capacidades de todos los alumnos.

Se comprende así la dificultad de establecer claramente la implementación de las TIC en un lugar destacado considerando el contexto propio de la modalidad. Entendemos que sería necesario que el docente trabaje para construir comunidades de aprendizaje y aprenda al establecer valores inclusivos abordando y respetando la diversidad. (Carreras, 2010)

Durante todo el trabajo que ha representado esta investigación la autora ha participado de varias propuestas pedagógicas con algún nivel de implementación de TIC de modo transversal al contenido curricular. Se ha podido observar en innumerables ocasiones la dificultad que presenta el docente para el uso de las TIC como recursos transversales y no como una actividad aislada del contexto de la clase. Pero también se ha observado a docentes que aunque muy superficiales en sus conocimientos relacionados con las tecnologias han invertido tiempo y deseos de formación para apropiarse del uso pedagógico de las TIC llevando a buen puerto diversos proyectos junto a sus alumnos/as. Los/as docentes en general se están abriendo a ser asesorados por sus alumnos en recursos que le son poco familiares. Las escuelas, como instituciones, también se están habituando a esta dinámica en la cual el alumno es co-constructor con el docente de los proyectos que van 
surgiendo. De este modo el alumno y el docente están flexibilizando sus roles, un oficio de alumno y un oficio de docente actuando en colaboración a modo de comunidad de prácticas para sumergirse en el mundo digital en favor de un aprendizaje constructivoy significativo para ambos actores.

De las principales conclusiones de la investigación y en relación con nuestros interrogantes iniciales, podemos expresar.

Cuando hay relación entre la estrategia didáctica, el contenido y los aprendizajes que se promueven en función de la discapacidad o problemática específica se puede entender que las tecnologías favorecen la comprensión y apropiación de estos saberes.

Atravesar los contenidos curriculares por medio de los entornos digitales permitiría ampliar las posibilidades de pensar (Lion, 2006) y estimular la construcción de modo reflexivo de soluciones frente a diversos problemas (Willson, 1998, citado en Lion, 2006).

En ocasiones es la discapacidad la que lleva al despliegue de determinada estrategia para favorecer el desenvolvimiento o fortalecer determinada competencia que es necesaria desplegar en el alumno o alumna. Son docentes que, por tanto, comprenden la interface necesaria para el abordaje de la discapacidad con herramientas tecnológicas pertinentes, tal como hemos analizado en el despliegue de las estrategias y de las competencias. 


\section{Referencias}

Angeloff, C., Muratore, F., Campo, A. y Savo, M. (2015). Vincul-Arte a un clic. Portal educativo Integrar. http:/ /integrar.bue.edu.ar/integrar/blog/articulo/vincularte-a-un-clic/

Baldissera, A. La escuela infernal. (2016). Portal educativo Integrar. http://integrar.bue.edu.ar/integrar/blog/articulo/la-escuela-infernal-2/

Buckingham, D. (2012). Más allá de la tecnología: aprendizaje infantil en la era digital. Manantial.

Burbules, N. (2011). Entrevista a Nicholás Burbules en Gvirtz, S. y Necuzzi, C., Educación y tecnologías: las voces de los expertos. (1a ed.). ANSES. http://www.oei.es/conectarigualdad.pdf

Buckingham, D. (2006). La educación para los medios en la era de la tecnología digital. (Ponencia). Congre so del décimo aniversario de MED "La sapienza di comunicare". Instituto para la educación. Universidad de Londres.

Carreras, J. (2010). Escuelas inclusivas. Un camino para construir entre todos. Investigación y relato de experiencias sobre educación y diversidad. Fundación Par.

Castellano, R. E. \& Sánchez Montoya, R. (2011). Laptop, andamiaje para la Educación Especial. Guía Práctica. Computadoras móviles en el currículum. UNESCO http://unesdoc.unesco.org/images/0021/002120/212091s.pdf

Coll, C. (2008). Aprender y enseñar con las TIC: expectativas, realidady potencialidades. Portal educ.ar. http://www.educ.ar/sitios/educar/recursos/ver?id=70819

DGCyE. (2008). Comisión TES. Dirección de Educación Especial.

http://servicios.abc.gov.ar/lainstitucion/sistemaeducativo/educacionespecial/caracterizacio nes/comision_tes.pdf

Echeverría, J. (2000). Educación y tecnologías telemáticas. Revista Iberoamericana de Educación, 24. DOI: $10.35362 /$ rie 240995.

Fainblum, A. (2004). Discapacidad. Una perspectiva desde el psicoanálisis. Editorial Tekne.

Glaser, B. y A. Strauss (1967). The discovery of grounded theory: estrategies for qualitative re search. Aldine Publishing Company.

Gobierno de la Ciudad de Buenos Aires Subsecretaría de Educación (2004) Diseño curricular para la escuela primaria: Segundo ciclo de la escuela primaria/educación general básica, tomo 1. La subsecretaría. Existencias: Diseño Curricular para la escuela primaria (me.gov.ar)

Grané, M. Entrevista a Mariona Grané en Gvirtz, S. y Necuzzi, C. (2011). Educación y tecnologias: las voces de los expertos. (1.a ed.). ANSES. http://www.oei.es/conectarigualdad.pdf

Guijarro, R. B. (1990). La atención a la diversidad en el aula y las adaptaciones del currículum. http://dialnet.unirioja.es / servlet/articulo?codigo=2089575

Liguori, L. (2000). Las nuevas tecnologías de la información y la comunicación en el marco de los viejos problemas y desafios educativos. En Litwin. E. (Comp.), Tecnología educativa. Política, historia, propuestas (pp.123-150). Paidós.

Lion, C. (2006). Imaginar con tecnologías. Relaciones entre tecnologías y conocimiento. La Crujía

Litwin, E. (2009). Tecnologias educativas en tiempos de internet. Paidós.

Maggio, M. (2012). Enriquecer la enseñanza. Los ambientes con alta disposición tecnológica como oportunidad. Paidós. 
Piaget, J. (2005). Inteligencia y afectividad. Aique.

Roma, M. C. (2013). La historieta. Integrar. Recursos pedagógicos. La historieta | Integrar (bue.edu.ar)

Roma, M. C. (2014) Blog escolar. Portal educativo Integrar. http://integrar.bue.edu.ar/integrar/blog/articulo/blog-escolar/

Roma, M. C. (2015). El texto biográfico. La biografia de Neymar. Integrar. Recursos pedagógicos. El texto biográfico: La biografia de Neymar | Integrar (bue.edu.ar)

Roma, M. C. (2018). Estrategias didácticas mediadas por las tecnologías de la información y la comunicación (TIC) en alumnos y alumnas de nivel primario con necesidades educativas especiales. ttp://catalogo.filo.uba.ar/cgi-bin/koha/opac-de tail.pl?biblionumber $=422582$

Sánchez Montoya, R. (2007). Capacidades visibles, tecnologías invisibles: Pe rspectivas y estudio de casos. Comunicación y pedagogía: Nuevas tecnologías y recursos didácticos, 220, pp. 32-38. http://dialnet.unirioja.es/servlet/articulo?codigo=2333719

Savo, M. (2015). Nos comunicamos por Edmodo. Portal educativo Integrar. http://integrar.bue.edu.ar/integrar/blog/articulo/nos-comunicamos-conedmodo/?ti po=Mis\%20Publicacione s\&usr $=2024$

Vasilachis De Gialdino, I. (1992). Tesis 7. En Métodos cualitativos I, Los problemas teóricoepistemológicos. Centro Editor de América Latina. 